\title{
Arbeitssicherheit: eine Notwendigkeit
}

\section{Michel Matter}

Dr., Vizepräsident der FMH, Departementsverantwortlicher Dienstleistungen und Berufsentwicklung

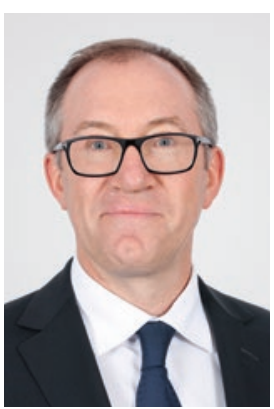

Das Jahr 2020 wird lange im kollektiven Gedächtnis bleiben. Zum Zeitpunkt, zu dem ich diese Zeilen zu Papier bringe, weiss niemand, wie und wann die gesundheitliche, soziale und wirtschaftliche Krise rund um das Coronavirus enden wird. Alles ist auf den Kopf gestellt, viele Gewissheiten sind verschwunden. Wir alle sind im beruflichen und privaten Leben betroffen. Den Angehörigen der Gesundheitsberufe wurde an vielen Abenden von ganzem Herzen applaudiert, um ihrer Arbeit Anerkennung zu zollen. Wir werden ihren Willen und ihre Entschlossenheit angesichts des unsichtbaren Feindes nicht vergessen. An vorderster Front im Kampf gegen die Pandemie arbeiteten sie Stunde um Stunde; zugleich mussten viele sich mit neuen Aufgaben vertraut machen und auf ein neues Arbeitsumfeld einstellen. Das Thema Arbeitssicherheit hat somit noch an Bedeutung gewonnen.

Nur ein interprofessioneller Blick ermöglicht es uns, die Realitäten und Anforderungen vor Ort zu verstehen.

Derzeit steigt die Zahl der durchgeführten Tests ebenso wie die der positiven Fälle immer weiter an. Und so sicher wie im Herbst die Blätter zu Boden schweben und wie die Krankenkassenprämien steigen, wird die saisonale Grippe kommen - mit Symptomen, die denen des Coronavirus ähneln. Sollte die zweite Welle so stark sein wie die erste, wäre dies für unsere Teams verheerend. Wir müssen alles dafür tun, um sämtliche Angehörigen der Gesundheitsberufe und der zugehörigen Verwaltungen auf physischer wie psychischer Ebene zu schützen, sei es in den Spitälern, in der ambulanten Versorgung oder in medizinischen Zentren und Praxen.

Im Januar 2019 wurde die FMH von mehreren kantonalen Ärztegesellschaften zum Thema Arbeitssicherheit befragt. Es geht darum, eine Branchenlösung auf nationaler Ebene zu finden. Die Vorgaben der Eid- genössischen Koordinationskommission für Arbeitssicherheit machen eine Antwort erforderlich, die in sämtlichen Arztpraxen anwendbar ist - und dabei die medizinischen Standards der einzelnen Kantone berücksichtigt. Die $\mathrm{H}+$ angeschlossenen Spitäler verfügen bereits über eine Branchenlösung. Auf Beschluss des Zentralvorstands der FMH und unter der Leitung meiner Mitarbeiterin Sonia Barbosa, der ich meine Anerkennung für die hohe Qualität der geleisteten Arbeit ausspreche, machte sich das Departement Dienstleistungen und Berufsentwicklung sofort an die Arbeit, um das Projekt einer Branchenlösung für Arztpraxen zu konkretisieren. Dabei wurden nicht nur die fachlichen Aspekte berücksichtigt, sondern auch die Komplexität und Besonderheiten der einzelnen Fachgebiete. Zugleich galt es, unnötige Zwänge und Kosten zu vermeiden. Es wurde eine interprofessionelle Expertengruppe einberufen, die sowohl die medizinischchirurgischen Fachrichtungen als auch alle Regionen des Landes vertritt. Nur ein interprofessioneller Blick ermöglicht es uns, die Realitäten vor Ort sowie die alltäglichen Gewohnheiten und Anforderungen jedes und jeder Einzelnen zu verstehen und zu kennen. In den Praxen ist der Gesundheitsschutz für das medizinische, paramedizinische und administrative Personal sowie für sämtliche Patientinnen und Patienten und ihre Angehörigen eine Notwendigkeit.

Die Arbeitssicherheit auf physischer wie psychischer Ebene muss höchste Priorität geniessen.

Die mit dem Coronavirus einhergehende Krise wirkte dabei als Katalysator und hat uns unsere nationalen und kantonalen gesetzlichen Verpflichtungen in Erinnerung gerufen. Somit ermöglicht die Branchenlösung es uns allen, einer Selbstverständlichkeit gerecht zu werden: Die Arbeitssicherheit auf physischer wie psychischer Ebene muss höchste Priorität geniessen. 